\title{
A NORMATIZAÇÃO DOS FITOTERÁPICOS NO BRASIL
}

\section{BRAZILIAN PHYTOTHERAPIC REGULAMENTATION}

\author{
OLIVEIRA, Andrezza Beatriz; LONGHI, Joy Ganem¹; ANDRADE, Cláudia \\ Alexandra ${ }^{1}$; MIGUEL, Obdúlio Gomes ${ }^{1,2}$ MIGUEL, Marilis Dallarmi ${ }^{1,3}$ \\ ${ }^{1}$ Programa de Pós Graduação em Ciências Farmacêuticas UFPR; ${ }^{2}$ Professor Adjunto de Fitoquímica do \\ Curso de Farmácia UFPR; ${ }^{3}$ Professor Adjunto de Farmacotécnica do Curso de Farmácia UFPR \\ *Autor para envio de correspondências: Programa de Pós-Graduação em Ciências Farmacêuticas - UFPR. Avenida \\ Prefeito Lothário Meissner, 632, Jardim Botânico, CEP 80210-170, Curitiba-PR: e-mail: dallarmi@ufpr.br \\ RECEBIDO: 07106 ACEITO: 12/07
}

\section{RESUMO}

A utilização de fitoterápicos tem apresentado um notável crescimento, exigindo uma normatização adequada para garantir a qualidade em todas as etapas de elaboração destes medicamentos. Neste trabalho foram relacionadas as legislações específicas pertinentes a cada etapa do seu desenvolvimento, incluindo a pesquisa científica, cultivo vegetal, produção do fitoterápico, controle de qualidade e registro frente aos órgãos competentes.

Palavras-chave: fitoterápico, normatização no Brasil.

\section{ABSTRACT}

The use of phytotherapic has presented an important increasing, demanding a regulamentation to guarantee the quality in all the stages of elaboration of these medicines. In these article, the specific laws to each step of its development had been related, inclunding cientific research, vegetable culture, phytotherapic production, quality control and register submited to the competent agencies.

Key-words: phytotherapic, brazilian regulamentation

\section{INTRODUÇÃO}

O conhecimento sobre plantas medicinais simboliza muitas vezes o único recurso terapêutico de muitas comunidades e grupos étnicos, sendo que o uso de plantas no tratamento de enfermidades é tão antigo quanto a espécie humana (MACIEL, PINTO, VEIGA JR, 2001).

Os primeiros relatos do uso das plantas medicinais datam de 2.600 a.C. tais espécies utilizadas na Mesopotâmia ainda são mencionadas pelas sociedades atuais para tosse, febre e inflamação (GURIB - FAKIM, 2006) 
Fitoterápico, em um conceito bastante amplo e constante em legislação (Portaria 06/95 ANVISA) pode ser definido como "Todo medicamento tecnicamente obtido e elaborado, empregando-se, exclusivamente, matérias primas ativas vegetais com a finalidade profilática, curativa ou para fins de diagnóstico, com benefício para o usuário. É caracterizado pelo conhecimento da eficácia e dos riscos de seu uso, assim como pela reprodutibilidade e constância de sua qualidade; é o produto final acabado, embalado e rotulado. $\mathrm{Na}$ sua preparação podem ser utilizados adjuvantes farmacêuticos permitidos pela legislação vigente. Não podem estar incluídas substâncias ativas de outras origens, não sendo considerado produto fitoterápico quaisquer substâncias ativas, ainda que de origem vegetal, isoladas ou mesmo suas misturas." (BRASIL, 1995).

Tendo em vista que a maior parte da população mundial ocupa os países menos desenvolvidos economicamente, os quais encontram dificuldades em oferecer atendimento à saúde frente ao aumento populacional, escassez de recursos e necessidade aumentada de medicamentos que se tornam mais dispendiosos, destaca-se a importância da Fitoterapia (WIJESEKERA, 1986).

O estímulo ao uso de fitoterápicos objetiva prevenir, curar ou minimizar os sintomas das doenças, com um custo mais acessível à população e aos serviços públicos de saúde, comparativamente àqueles obtidos por síntese química, que são, em geral, mais caros, devido às patentes tecnológicas envolvidas (TOLEDO et al; 2003).

Segundo a Organização Mundial de Saúde, o uso de plantas medicinais para o tratamento das mais diversas enfermidades tem levado países como a China a consumir dentre o total de medicamentos cerca de $30-50 \%$ de drogas de origem vegetal. Na Europa e América do Norte $50 \%$ da população já fez uso de fitoterápicos. Na Alemanha destaca-se um índice de $90 \%$ de uso de formas farmacêuticas contendo algum componente de origem vegetal (WHO, 2003).

Sendo assim, a Fitoterapia constitui uma forma de terapia medicinal em notável crescimento, cujo mercado mundial movimenta em torno de 22 bilhões de dólares no qual o Brasil representa apenas cerca de 23\% (YUNES, PEDROSA, CECHINEL FILHO, 2000). As estimativas nacionais apontam que $82 \%$ da população brasileira utiliza produtos à base de ervas, e o setor 
fitoterápico conta com duzentas empresas e movimenta um bilhão de reais em toda sua cadeia produtiva e emprega mais de cem mil pessoas no país (ABIFITO, 2006).

A utilização de plantas medicinais tem recebido incentivos da Organização Mundial de Saúde, mediante a Resolução WHA 31.33 (1978) e 40.33 (1987), que reafirmam a importância das plantas medicinais nos cuidados com a saúde, recomendando entre outros aspectos a criação de programas globais para a identificação, validação, preparação, cultivo e conservação das plantas medicinais utilizadas na medicina tradicional, bem como assegurar o controle de qualidade dos fitoterápicos (MIGUEL; MIGUEL, 1999).

Diante disto, o Ministério da Saúde baixou Diretrizes e Prioridades de Investigação em Saúde (Portaria 212, de 11/09/1981), incluindo as plantas medicinais. Em 1988, a Comissão Nacional Interministerial de Planejamento e Coordenação (CIPLAN) implanta a Fitoterapia como prática oficial da medicina e orienta sua inclusão nos serviços primários de saúde. Esta recomendação condiciona o uso de plantas medicinais a estudos científicos, que incluem a investigação antropológica-botânica junto à medicina popular tradicional popular; isolamento e caracterização de substâncias ativas; transformação química gerando novos fármacos, ensaios farmacológicos, toxicológicos, préclínicos e clínicos (MIGUEL; MIGUEL, 1999). O Decreto 5813 (MCT), de 22/06/2006, enfatiza esta prioridade ao aprovar a Política Nacional de Plantas Medicinais e Fitoterápicos.

No contexto da utilização de fitoterápicos faz-se importante a existência de normatização a ser cumprida com a finalidade de garantir a qualidade destes medicamentos, de forma a possibilitar um adequado tratamento das enfermidades, garantindo o cumprimento do direito constitucional do cidadão conforme o Art. 196 da Constituição da República Federativa do Brasil "A saúde é direito de todos e dever do Estado" (BRASIL, 1988).

Para tanto, a transformação de uma matéria-prima vegetal em um medicamento deve visar à preservação da integridade química e farmacológica da planta, garantindo a constância de sua ação biológica e a segurança de sua utilização, além de valorizar seu potencial terapêutico. Para garantir esses objetivos, a pesquisa para o desenvolvimento de fitoterápicos inclui várias 
etapas envolvendo um processo interdisciplinar, multidisciplinar e interinstitucional (MIGUEL, MIGUEL, 1999).

\section{ETAPAS DE ELABORAÇÃO DE FITOTERÁPICOS E A REGULAMENTAÇÃO}

As etapas de desenvolvimento de fitoterápicos envolvem diversas áreas do conhecimento, como a etnobotânica, botânica, agronomia, ecologia, química, fitoquímica, farmacologia, toxicologia, biotecnologia e tecnologia farmacêutica (TOLEDO et al., 2003).

\subsection{Pesquisa científica}

O desenvolvimento de fitoterápicos inicia-se por pesquisa científica, que perpassa por levantamento bibliográfico e ensaios práticos que buscam resultados quanto a aspectos agronômicos, fitoquímicos, e avaliam a atividade biológica do vegetal (MIGUEL, MIGUEL, 1999).

O levantamento bibliográfico em literatura científica, associado aos estudos etnobotânicos (quais tratam da observação do uso popular de plantas nas diferentes culturas) el ou quimiotaxonômicos (envolvendo o aspecto morfológico para revelar a presença de determinados grupos químicos que possuam atividade famacológica), bem como registros de toxicidade no emprego do vegetal avaliado. Submete-se então a planta escolhida para avaliação das características macroscópicas e morfoanatômicas, necessárias tanto para a identificação botânica do vegetal como para o controle de qualidade.

Tais aspectos são regulamentados por legislações específicas:

- PN 174P (IBDF), de 11/03/1981: estabelece a autorização especial para pesquisas com plantas em Parques Nacionais e Reservas Biológicas Federais;

- D.98830 (PR), de 15/01/1990: referencia sobre a coleta, por estrangeiros, de dados e materiais científicos, no Brasil;

- Port. 40 (MS), de 10/01/1994: nomeia a Subcomissão da Comissão Permanente da Farmacopéia Brasileira para elaborar monografias de plantas medicinais; 
- Port. 42 (MS), de 10/01/1994: nomeia a Subcomissão da Comissão Permanente da Farmacopéia Brasileira para elaborar normas sobre fitoterápicos;

- Port. 175 (MS), de 26/01/1996: aprova o fascículo I da Parte II da 4a. Edição da Farmacopéia Brasileira, contendo 30 textos revisados de edições anteriores e 72 novos textos;

- Port. 116 (SVS), de 22/11/1995: indica Farmacopéias aceitas no Brasil;

- L.9279 (PR), de 14/05/1996: Lei de patentes;

- Port. 90 (SVS), de 13/06/1996: apresenta proposta de lista de fármacos, plantas medicinais e adjuvantes de tecnologia farmacêutica que serão incluídos no fascículo 2 da Parte II da Farmacopéia Brasileira IV;

- Res. 196 (CNS), de 10/10/1996: aprova diretrizes e normas regulamentadoras para pesquisas envolvendo seres humanos;

- RDC 48 (ANVISA), de 16/03/2004: para esta etapa, determina a apresentação de levantamento bibliográfico etnofarmacológico e de utilização, documentações técnico-científicas ou publicações.

- Lei 12259 (PR), de 23/08/2002: dispõe sobre a política estadual de incentivo à pesquisa e a preparação de produtos fitoterápicos, com o objetivo de facultar ao Sistema Único de Saúde - SUS - o uso desses medicamentos no tratamento de determinadas enfermidades.

- Resolução RE 88 (ANVISA), de 16/03/2004: determina a publicação da "lista de referências bibliográficas para avaliação de segurança e eficácia de fitoterápicos".

- Port. 2311 (MCT), de 29/09/2006: institui o grupo de trabalho, composto por representantes dos órgãos relacionados, para elaborar o Programa Nacional de Plantas Medicinais e Fitoterápicos.

A etapa seguinte objetiva a produção abundante e homogênea de matéria-prima, preservando sua qualidade, a espécie e a biodiversidade sob aspectos ecológicos. Os estudos agronômicos são regulamentados por:

- DL 4720 (PR), de 21/09/1942: estabelece normas gerais para o cultivo de plantas entorpecentes e sua manipulação; 
- Port. 32 (SNVS), de 05/07/1986: distingue vegetais com fins alimentícios e medicamentosos;

- RDC 17 de 24/02/2000 (ANVISA): regulamenta que a matéria-prima para a produção de fitoterápicos deve ser isenta de contaminantes microbianos e biológicos, metais pesados e agrotóxicos.

- Instrução Normativa 09 (MAPA), de 02/06/2005: aprova as normas para produção, comercialização e utilização de sementes.

- Instrução Normativa 24 (MAPA), de 16/12/2005: aprova as normas para produção, comercialização e utilização de mudas.

Os estudos fitoquímicos, por sua vez, compreendem as etapas de isolamento, elucidação estrutural e identificação dos constituintes mais importantes do vegetal, responsáveis ou não pela ação biológica (marcadores químicos), permitindo identificar a espécie vegetal, bem como analisar e caracterizar frações ou substâncias bioativas. A normatização que descreve tal etapa é a RDC 48 de 16/03/2004 (ANVISA), que determina as informações a serem fornecidas sobre a droga e o derivado vegetal e o controle de qualidade.

Uma vez determinados os constituintes químicos da matéria-prima vegetal, realiza-se a avaliação da atividade biológica, qual inclui a investigação da atividade farmacológica e toxicológica dos extratos, frações ou das substâncias isoladas a partir da droga vegetal.

Regulamentam esta etapa:

- Resolução 196/96 e 251/97(CNS): exige a apresentação de estudos científicos que comprovem a segurança e eficácia do uso de medicamentos; - RDC 48 de 16/03/2004 (ANVISA): aprova o Regulamento Técnico, atualizando a normatização do registro de medicamentos fitoterápicos; estabelece que os medicamentos fitoterápicos registrados anteriormente a 31/01/1995, com exceção dos enquadrados como fitoterápicos tradicionais, devem apresentar, no primeiro protocolo de renovação de registro os relatórios de segurança e eficácia de produção e o controle de qualidade, atualizados de acordo com o Regulamento Técnico; 
- RE 88 de 16/03/2004 (ANVISA): determina a publicação da "Lista de Referências Bibliográficas para Avaliação de Segurança e Eficácia de Fitoterápicos".

- RE 89 de 16/03/2004 (ANVISA): determina a publicação da "Lista de Registro Simplificado de Fitoterápicos".

- RE 90 de 16/03/2004 (ANVISA): determina a publicação da "Guia para a Realização de Estudos de Toxicidade Pré-Clínica de Fitoterápicos";

- RDC 222, de 29/07/2005 (ANVISA): especificações de qualidade descritas na edição vigente da Farmacopéia Brasileira.

\subsection{Cultivo do vegetal}

Concluída a pesquisa científica, cujos resultados comprovem a segurança e a eficácia do vegetal para a sua utilização como medicamento fitoterápico, segue-se o cultivo da planta segundo os aspectos agronômicos determinados previamente e de acordo com a mesma normatização.

As instituições que promovem o cultivo da matéria-prima vegetal podem servir de fornecedores àquelas produtoras do medicamento fitoterápico.

\subsection{Produção e Controle de Qualidade dos Fitoterápicos}

Para a produção de fitoterápicos, além da realização prévia de todos os estudos anteriores, os procedimentos e etapas de processamento devem estar devidamente estabelecidos. A produção de medicamentos é etapa importante, regulamentada por:

- Port. 22 de 30/10/1967 (SNFMF): Normas para o emprego de preparações fitoterápicas;

- RDC 210 de 04/08/2003 (ANVISA): determina o cumprimento das diretrizes estabelecidas no Regulamento Técnico das Boas Práticas para a Fabricação de Medicamentos a todos os estabelecimentos fabricantes de medicamentos; institui e aprova a Classificação e Critérios de Avaliação dos itens constantes do roteiro de Inspeção para Empresas Fabricantes de Medicamentos; institui como norma de inspeção para fins de verificação do 
cumprimento das Boas Práticas de Fabricação de Medicamentos; relaciona que as empresas fabricantes de medicamentos devem proceder a autoinspeções, conforme o Regulamento Técnico das Boas Práticas para a Fabricação de Medicamentos e o Roteiro de Inspeção em Indústria Farmacêutica, como parte das medidas necessárias à implementação das mesmas;

- RDC 48 de 16/03/2004 (ANVISA): aprova o Regulamento Técnico, atualizando a normatização do registro de medicamentos fitoterápicos; determina que todos os testes referentes a controle de qualidade (quando terceirizados), deverão ser executados em instituições credenciadas no sistema REBLAS ou por empresas fabricantes de medicamentos que tenham Certificado de Boas Práticas de Fabricação atualizado e satisfatório;

- RDC 222, de 29/07/2005 (ANVISA): especificações de qualidade descritas na edição vigente da Farmacopéia Brasileira.

Uma vez produzido, o fitoterápico é embalado conforme determinação das normatizações RDC 222/05 (ANVISA), RDC 210/03 (ANVISA) e Portaria 519/98 (ANVISA). Tendo em vista que a elaboração de medicamentos é um processo amplo e com etapas críticas, a qualidade do fitoterápico só pode ser garantida quando o controle ocorre em paralelo às referidas etapas. Portanto, as normas já citadas mencionam procedimentos e especificações para o controle de qualidade.

A propaganda mercadista conforma-se à Lei 9294/96 (MAPA) e Decreto 2018/96 (MAPA), não deve levar a confusões de interpretação, deve indicar propriedades terapêuticas somente se comprovadas cientificamente assim como cuidados e contra-indicações, e referir-se apenas a produtos registrados frente ao órgão de vigilância sanitária competente.

\subsection{Registro frente ao órgão de vigilância sanitária competente}

O referido registro do fitoterápico é conferido somente após a comprovação do bom cumprimento de todas as etapas de elaboração, seguindo as normatizações RDC 48/04 (ANVISA), RDC 210/03 (ANVISA) já descritas, além da Resolução RE 91/04, que determina a publicação da "Guia 
para Realização de Alterações, Inclusões, Notificações e Cancelamentos PósRegistro de Fitoterápicos", estabelecendo a documentação e os ensaios exigidos pela ANVISA.

Portanto, a garantia do fornecimento de fitoterápicos com qualidade satisfatória para seu emprego eficaz como ferramenta terapêutica, conta com a legislação e fiscalização por órgãos competentes, além da responsabilidade e conscientização dos indivíduos envolvidos nas etapas de sua elaboração.

\section{CONSIDERAÇÕES}

Tendo em visa o amplo consumo de produtos de origem vegetal no Brasil e o crescente desenvolvimento de fitoterápicos como medicamentos em nível industrial, a aplicação de normas sobre a sua elaboração faz-se importante. Além disso, a regulamentação e oficialização do desenvolvimento e uso de fitoterápicos contrapõe a crença culturalmente fixada de que "o que é natural não possui efeitos colaterais". Tal afirmação é enganosa e remete a inúmeros riscos a saúde da população. Diversos estudos demonstram que as plantas medicinais possuem efeitos indesejáveis e muitas vezes tóxicos, remetendo à conscientização do seu bom uso, segundo aspectos também legislativos (TOLEDO et al., 2003).

A legislação de fitoterápicos brasileira é considerada altamente exigente em todo o mundo, justificando os esforços para garantir a sua qualidade. No entanto, ainda restam arestas a serem reparadas e dificuldades a serem vencidas.

Um grande fator limitante é que a maioria das plantas em uso não se encontra descrita em códigos oficiais (formulários e farmacopéias), não havendo inclusive estudos sobre as mesmas. Deve-se destacar ainda, que o desenvolvimento de um novo medicamento envolve um processo complexo de alto custo, onde se requer investimentos em torno de 100-360 milhões de dólares em um período de 10-12 anos para que um fármaco seja desenvolvido (YUNES, PEDROSA, CECHINEL FILHO, 2001). Isto tem levado as companhias farmacêuticas a aumentarem significativamente o interesse por pesquisas na área e sobretudo tem gerado um aumento no faturamento oriundo da comercialização de fitoterápicos nos EUA e Europa. 
Somam-se a estas limitações o grande número de pesquisas desvinculadas umas das outras, onde as especialidades se sobrepõem às reais necessidades da Fitoterapia enquanto medicina tradicional aplicada. Constatam-se inúmeras plantas cujos estudos fitoquímicos e farmacológicos são potencialmente satisfatórios, contudo a espécie em questão não possui sequer pesquisas agronômicas e ecológicas que se preocupam com reprodução do vegetal ou sequer o extrativismo sustentável. Nesta perspectiva, investir nos códigos oficiais cuja finalidade é a construção de monografias que privilegiem desde o cultivo, perpassando pelo manejo adequado, até chegar à determinação de doses clínicas poderá representar a solução para a indústria nacional e para os centros de produção institucionais, além de atender às necessidades básicas em saúde, e gerar empregos em áreas rurais e centros industriais (TOLEDO et al., 2003).

Por outro lado, a indústria nacional de fitoterápicos possui inúmeras drogas comercializadas sob forma não medicamentosa, com registro alimentício, com base somente no uso popular, sendo estas plantas alvos do interesse de indústrias farmacêuticas. Isto remete à necessidade de estudos fitoquímicos da atividade biológica, com vistas à eficiência e segurança terapêutica, para a comprovação científica e conseqüente produção lícita de medicamentos fitoterápicos, conforme exigências do registro frente ao órgão regulatório competente.

\section{CONCLUSÃO}

Com uma legislação atualizada e adequada, a indústria nacional será motivada a incentivar a pesquisa e o desenvolvimento tecnológico de produtos brasileiros de origem natural. Somente uma legislação justa irá assegurar à nossa população o acesso a produtos naturais eficazes e de excelente qualidade. Contudo, a legislação vigente para a produção de fitoterápicos não apresenta especificações bem definidas para evitar alterações quali e quantitativas nas substâncias ativas das plantas, devido ao cultivo em regiões geograficamente diferenciadas (fatores ecofisiológicos), bem como influências sazonais (chuvas, fotoperíodo), nutrição do solo, contaminação por fungos e bactérias, competição com outras plantas, período da coleta, como também os 
aspectos agronômicos de cultivo e manejo que geram a matéria-prima (droga) para a produção farmacêutica, assim como a armazenagem, transporte entre outros.

Portanto, verifica-se a necessidade e importância da revisão constante da normatização nacional para o desenvolvimento de fitoterápicos, incluindo maior detalhamento e aspectos específicos, sem deixar de oferecer praticidade quanto à consulta das normas, tornando-as diminuídas em número, reduzindo a possibilidade de incertezas. No exercício da regulamentação, cabe aos órgãos fiscalizadores incluir também as orientações de execução da lei.

\section{REFERÊNCIAS}

ABIFITO. Uma legislação justa para os produtos de origem natural. 2006. Disponível em: <http://www.abifito.com.br/introducao.asp>, acesso em: 18jun.2006.

BRASIL. Constituição da República Federativa do Brasil. Diário Oficial da União de 5 de Dezembro de 1988. Brasília. Disponível em: <http://www010.dataprev.gov.br/sislex/paginas/22/Consti.htm>, Consulta em: 5jul.2006.

BRASIL. Ministério da Agricultura, Pecuária e Abastecimento. Decreto n. 2018 de 01 de outubro de 1996. Diário Oficial da União de 02 de outubro de 1996. Brasília. Disponível em: <http://extranet.agricultura.gov.br/sislegisconsulta/consultarLegislacao.do;jsessionid=c0a8017ace6c369487ec28b4b79b5 61f09ca42bba63.e34Oah8PbhiKby0Lb30Pc3uKaxiQe6fznA5Pp7ftolbGmkTy>, Consulta em: 26jan.2006.

BRASIL. Ministério da Agricultura, Pecuária e Abastecimento. Instrução Normativa n. 9 de 02 de junho de 2005. Diário Oficial da União de 10 de junho de $2005 . \quad$ Brasília. Disponível em: <http://extranet.agricultura.gov.br/sislegisconsulta/consultarLegislacao.do;jsessionid=c0a8017ace6c369487ec28b4b79b5 61f09ca42bba63.e34Oah8PbhiKby0Lb30Pc3uKaxiQe6fznA5Pp7ftolbGmkTy>, Consulta em: 26jan.2006.

BRASIL. Ministério da Agricultura, Pecuária e Abastecimento. Instrução Normativa n. 24 de 16 de dezembro de 2005. Diário Oficial da União de 20 de dezembro de 2005. Brasília.

Disponível em: <http://extranet.agricultura.gov.br/sislegisconsulta/consultarLegislacao.do;jsessionid=c0a8017ace6c369487ec28b4b79b5 61f09ca42bba63.e34Oah8PbhiKby0Lb30Pc3uKaxiQe6fznA5Pp7ftolbGmkTy>, Consulta em: 26jan.2006.

BRASIL. Ministério da Agricultura, Pecuária e Abastecimento. Lei n. 9294 de 15 de julho de 1996. Diário Oficial da União de 15 de julho de 1996. Brasília. Disponível em: <http://extranet.agricultura.gov.br/sislegisconsulta/consultarLegislacao.do;jsessionid=c0a8017ace6c369487ec28b4b79b5 
61f09ca42bba63.e34Oah8PbhiKby0Lb30Pc3uKaxiQe6fznA5Pp7ftolbGmkTy>, Consulta em: 26 jan.2006.

BRASIL. Ministério da Ciência e Tecnologia. Decreto n. 2311 de 22 de junho de 2006. Diário Oficial da União de 22 de Junho de 2006. Brasília. Disponível em: $\quad$ http://portal.saude.gov.br/portal/arquivos/pdf/DECRETO\%20N\%C2\% BA5205813\%20_2_.pdf>, Consulta em: 20 dez. 2006.

BRASIL. Ministério da Ciência e Tecnologia. Portaria n. 2311 de 29 de setembro de 2006. Diário Oficial da União de 29 de Setembro de 2006. Brasília. Disponível em: <http://www.mct.gov.br/index.php/content/view/39787.htnl>, Consulta em: 20 dez. 2006.

BRASIL. Ministério da Saúde. Agência Nacional de Vigilância Sanitária (ANVISA). Portaria n. 6 de 31 de janeiro de 1995. Diário Oficial da União de 31 de Janeiro de 1995. Brasília. Disponível em: <http://www.anvisa.gov.br/elegis/>, Consulta em: 26jan.2006.

BRASIL. Ministério da Saúde. Agência Nacional de Vigilância Sanitária (ANVISA). Resolução da Diretoria Colegiada RDC 48 de 16 de março de 2004. Diário Oficial da União de 18 de Março de 2004. Brasília. Disponível em: <http://www.anvisa.gov.br/e-legis/>, Consulta em: 26jan.2006.

BRASIL. Ministério da Saúde. Agência Nacional de Vigilância Sanitária (ANVISA). Resolução RE 88 de 16 de março de 2004. Diário Oficial da União de 18 de Março de 2004. Brasília. Disponível em: <http://www.anvisa.gov.br/elegis/>, Consulta em: 26jan.2006.

BRASIL. Ministério da Saúde. Agência Nacional de Vigilância Sanitária (ANVISA). Resolução RDC 17 de 24 de fevereiro de 2000. Diário Oficial da União de 25 de fevereiro de 2000. Brasília. Disponível em: <http://www.anvisa.gov.br/e-legis/>, Consulta em: 26jan.2006.

BRASIL. Ministério da Saúde. Agência Nacional de Vigilância Sanitária (ANVISA). Resolução RE 89 de 16 de março de 2004. Diário Oficial da União de 18 de março de 2004. Brasília. Disponível em: <http://www.anvisa.gov.br/elegis/>, Consulta em: 26jan.2006.

BRASIL. Ministério da Saúde. Agência Nacional de Vigilância Sanitária (ANVISA). Resolução RE 90 de 16 de março de 2004. Diário Oficial da União de 18 de março de 2004. Brasília. Disponível em: <http://www.anvisa.gov.br/elegis/>, Consulta em: 26jan.2006.

BRASIL. Ministério da Saúde. Agência Nacional de Vigilância Sanitária (ANVISA). Resolução RDC 222 de 29 de julho de 2005. Diário Oficial da União de 15 de agosto de 2005. Brasília. Disponível em: <http://www.anvisa.gov.br/e-legis/>, Consulta em: 26jun.2006.

BRASIL. Ministério da Saúde. Agência Nacional de Vigilância Sanitária (ANVISA). Resolução RDC 210 de 04 de agosto de 2003. Diário Oficial da União de 14 de agosto de 2003. Disponível em: <http://www.anvisa.gov.br/elegis/>, Consulta em: 26jan.2006.

BRASIL. Ministério da Saúde. Agência Nacional de Vigilância Sanitária (ANVISA). Portaria n. 519 de 26 de junho de 1998. Diário Oficial da União de 29 de junho de 1998. Brasília. Disponível em: <http://www.anvisa.gov.br/elegis/>, Consulta em: 26jan.2006.

BRASIL. Ministério da Saúde. Agência Nacional de Vigilância Sanitária (ANVISA). Resolução RE 91 de 16 de março de 2004. Diário Oficial da União 
de 18 de março de 2004. Brasília. Disponível em: <http://www.anvisa.gov.br/elegis/>, Consulta em: 26jun.2006.

GURIB-FAKIM, A. Medicinal plants: traditions of yesterday and drugs of tomorrow. Molecular Aspects of Medicine, v. 27, p. 1-93, 2006.

MACIEL, M. A. M.; PINTO, A. C.; VEIGA JR, V. F. Plantas medicinais: a necessidade de estudos multidisciplinares. Química Nova, v. 25, n. 3, 2002.

MIGUEL, M. D.; MIGUEL, O. G. Desenvolvimento de fitoterápicos. São Paulo : Robe, 1999.

TOLEDO, A. C.; HIRATA, L. L.; BUFFON, M. C. M.; MIGUEL, M. D.; MIGUEL, O. G. Fitoterápicos: uma abordagem farmacotécnica. Revista Lecta, v. 21, n. 1/2, p. 7-13, 2003.

WHO 2003. Traditional medicine. Disponível em: <http://www.who.int/mediacentre/factsheets/fs134/en/>, Consulta em: 20 dez.2006.

WIJESEKERA, R. O. B. Plant-derived medicines and their role in global health. The medicinal Plant Industry.1986.

YUNES, R. A.; PEDROSA, R. C.; CECHINEL FILHO, V. Fármacos e fitoterápicos: a necessidade do desenvolvimento da indústria de fitoterápicos e fitofármacos no Brasil. Química Nova, v. 24, n. 1, 2001. 